\title{
Rapid picture processing: Affective primes and targets
}

\author{
TOBIAS FLAISCH, ${ }^{\mathrm{a}, \mathrm{b}}$ MARKUS JUNGHÖFER, ${ }^{\mathrm{a}, \mathrm{c}}$ MARGARET M. BRADLEY, \\ HARALD T. SCHUPP, ${ }^{a}$ AND PETER J. LANG ${ }^{b}$ \\ ${ }^{a}$ Department of Psychology, University of Konstanz, Konstanz, Germany \\ ${ }^{\mathrm{b}}$ Center for the Study of Emotion and Attention, University of Florida, Gainesville, Florida, USA \\ ${ }^{c}$ Institute for Biomagnetism and Biosignalanalysis, Münster University Hospital, Münster, Germany
}

\begin{abstract}
In rapid serial visual presentation of pictures, an early ERP component shows enlarged negativity over occipital regions for emotional, compared to neutral, pictures. The present study examined the processing of emotional target pictures as a function of the hedonic valence of a preceding prime picture. Dense sensor ERPs were measured while subjects viewed a continuous stream of pleasant, neutral, and unpleasant pictures, presented for $335 \mathrm{~ms}$ each. Two main findings were observed: First, replicating previous results, emotional target pictures were associated with a larger posterior negativity, compared to neutral pictures. Second, the emotional content of the preceding prime picture affected target processing, with pleasant or unpleasant primes resulting in reduced negativity of the following picture, irrespective of its emotional valence. These findings are discussed within a framework of competition among successive pictures.
\end{abstract}

Descriptors: Attention, Emotion, ERP, Posterior negative potential, Pictures, Interference

Event-related potentials (ERPs) measured during picture viewing suggest selective processing of both pleasant and unpleasant, compared to neutral, stimuli. Among the ERP components modulated by picture emotionality is a late positive potential (LPP), measured over centro-parietal sensors that is enhanced when viewing emotionally arousing, compared to neutral, pictures (e.g., Cuthbert, Schupp, Bradley, Birbaumer, \& Lang, 2000; Schupp, Junghöfer, Weike, \& Hamm, 2003). Picture emotionality also affects ERPs in an earlier time window: When pictures are presented very rapidly, for instance, emotional pictures prompt enhanced negativity over occipital sensors in a window beginning approximately $150 \mathrm{~ms}$ after onset (early posterior negativity [EPN]; Junghöfer, Bradley, Elbert, \& Lang, 2001; Schupp et al., 2003). Both functional and evolutionary factors favor the selective processing of cues that help to facilitate the survival of the individual and the species. Thus, according to a motivational model of emotion (Lang, Bradley, \& Cuthbert, 1997), selective processing of pleasant and unpleasant cues reflects the inherent salience of these stimuli, which are important for implementing actions to stimuli that can sustain (appetitive) and threaten (defensive) the life of the organism.

In the rapid serial visual presentation (RSVP) paradigm, one hallmark of selective processing is a lingering effect of processing a salient target on stimuli that are subsequently presented. Thus,

This research was supported by grants from the German research foundation Schu1074/7-3 and Schu1074/10-1 to Harald Schupp. We thank Rafael Jódar for his assistance in data collection.

Address reprint requests to: Tobias Flaisch, Department of Psychology, University of Konstanz, P.O. Box D36, 78457 Konstanz, Germany. E-mail: Tobias.Flaisch@uni-konstanz.de when participants are asked to report two stimuli in a rapidly presented stream, report of the second stimulus is impaired when it is presented within a few hundred milliseconds of another target (Intraub, 1999; Potter, 1999). If the enhanced negativity found when viewing emotional pictures in the RSVP paradigm indicates selective processing, one hypothesis is that processing of subsequent pictures should be affected. Support for this hypothesis comes from a recent study that presented both emotional and neutral pictures in a RSVP stream (Most, Chun, Widders, \& Zald, 2005). When a neutral target stimulus followed the presentation of an emotional, compared to a neutral, picture, performance was impaired, despite the fact that explicit detection of the emotional picture was not required. Because affective pictures impaired subsequent performance in a manner similar to explicit targets, these emotional cues appear to naturally engage selective processing. Further evidence that affective stimuli are processed like explicit (neutral) targets is the widespread finding of a similar enhanced posterior negativity early in the processing window when subjects process stimuli to which they were instructed to selectively attend (Codispoti, Ferrari, Junghöfer, \& Schupp, 2006b; Potts \& Tucker, 2001; Schupp et al., 2007b).

In the present study, we assessed whether selective processing, as indicated by early occipital negativity in the RSVP paradigm, affects ERPs to subsequent pictures. A secondary aim was to replicate previous RSVP findings. Drawing on the data of Most et al. (2005) and other data suggesting that affective pictures naturally engage selective processing (Lang et al., 1997), participants simply viewed a set of affective and neutral pictures in the absence of an explicit task. Thus, pleasant, neutral, and unpleasant pictures were presented in a rapid serial presentation paradigm containing sequences that varied the hedonic content of the 


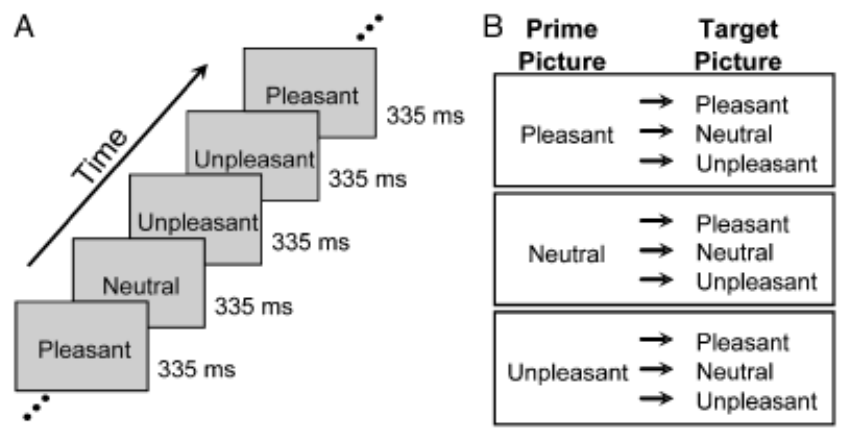

Figure 1. A: Illustration of the rapid serial visual presentation paradigm. Pleasant, neutral, and unpleasant pictures were presented for $335 \mathrm{~ms}$ without perceivable interstimulus gap. B: Illustration of the critical prime-target picture pairs examined in the present study.

current and subsequent pictures over the course of the experiment. Altogether, 6000 stimuli were presented $(300$ pictures $\times 20$ repetitions) for $335 \mathrm{~ms}$ each (with no interpicture interval) in a rapid stream. Previous studies have established that stimulus repetition does not eliminate selective processing as indicated by posterior negativity or the LPP (Codispoti, Ferrari, \& Bradley, 2006a, 2007; Schupp et al., 2006), allowing multiple presentations of the same picture. The focus of the analysis was on ERPs to contiguous pairs of pictures in which the emotionality (pleasant, neutral, unpleasant) of each picture in the pair was systematically covaried (see Figure 1).

Using this design, we expected to replicate previous effects showing greater posterior negativity over occipital sensors in an early time window when emotional (pleasant and unpleasant), compared to neutral, pictures were the focus of current processing. This pattern supports an interpretation that the early occipital negativity reflects the enhanced processing of the current picture (Junghöfer et al., 2001; Schupp et al., 2003). The main question was to determine whether emotionality of a preceding picture impacts subsequent picture processing. Based on data from attentional blink studies (Chun \& Potter, 1995; Shapiro, Arnell, \& Raymond, 1997), one hypothesis is that when an emotional picture is presented shortly before another picture, processing of the subsequent picture is impaired. If posterior negativity reflects current processing, it may be hypothesized that posterior negativity should be reduced when a picture is preceded by an emotionally arousing picture. Statistically, these considerations predict a main effect of picture emotionality for each picture in a pair. On the one hand, emotional pictures that are the current focus of processing are expected to be associated with greater posterior negativity compared to neutral images. And, on the other hand, affective pictures are expected to reduce the posterior negativity of subsequent pictures (regardless of emotionality).

The design of this study also naturally suggests potential effects of "affective priming," in which ERPs might vary as a function of whether the two contiguous stimuli are similar or different in hedonic valence (e.g., both pleasant or both unpleasant). Although congruence in hedonic valence between two successive pictures has no special status in a motivated attention framework, in which selective processing is proposed to be similarly heightened for both pleasant and unpleasant stimuli (Lang et al., 1997), such effects might be expected from an affective priming perspective. In affective priming tasks, faster evaluation of targets is found when they are preceded by a short-duration (e.g., $300 \mathrm{~ms}$ ) prime of the same hedonic valence (Avero \& Calvo,
2006; Klauer \& Musch, 2003). It is less clear what type of modulation to expect specifically for ERPs if affective priming occurs for contiguous same-valenced pairs. If one assumes that early occipital negativity reflects enhanced stimulus processing, one version of an affective priming hypothesis predicts an interaction between the emotionality of pictures in a pair due to the facilitated processing of pictures that are preceded by hedonically similar pictures.

\section{Method}

\section{Participants}

Participants were 24 healthy adults (13 women); 22 received course credit for an introductory psychology class at the University of Florida and 2 were post-graduate laboratory employees. Participants were between the ages of 18 and 22 years $(M=19.0)$. Prior to the experiment, signed letters of consent conforming to institutional guidelines for human research were obtained from all participants.

\section{Stimulus Materials and Procedure}

Participants viewed 300 pictures $^{1}$ from the International Affective Picture System (IAPS; Lang, Bradley, \& Cuthbert, 2005), a collection of standardized photographic materials. The pleasant picture contents comprised erotica $(n=54)$ and scenes of sports and adventure $(n=33)$, with the remaining stimuli depicting scenes of various categories, such as landscapes or food. The neutral contents included images depicting people $(n=37)$, household objects $(n=30)$, and other neutral scenes (landscapes, buildings, mushrooms, animals, etc.). The unpleasant images were comprised of scenes of threat and violence $(n=51)$, injury and mutilation $(n=33)$ and accidents, disgusting contents, and aversive medical situations. The three hedonic categories differed significantly from each other in normative ratings of pleasure (range of 1-9; pleasant: $M=7.0, S D=0.6$; neutral: $M=5.2$, $S D=0.6$; unpleasant: $M=2.4, S D=0.7)$. Mean arousal levels for emotional categories were significantly higher than for neutral contents (pleasant: $M=6.2$, neutral: $M=3.1$, unpleasant: $M=6.4$, all $S D$ s $=0.6$ ).

Pleasant, neutral, and unpleasant pictures were presented in a continuous stream without perceivable interstimulus interval for $335 \mathrm{~ms}$ each (see Figure 1a). The entire picture set was repeated 20 times, resulting in a total of 6000 picture presentations. Several constraints were imposed on the stimulus order, assuring adequate control of sequence effects. The nine experimental cells (see Figure 1b) were controlled with an average frequency of 667 occurrences for each transition. Furthermore, sequences containing three to seven consecutive same valence pictures occurred roughly with the same respective frequencies. Finally, the entire picture set was presented before the picture series was presented again in a different order. Each participant viewed the same order of picture presentation.

Using Presentation software (Neurobehavioral Systems, Inc., Albany, CA), the pictures were shown on a 21-in. CRT monitor (75-Hz refresh rate) located approximately $100 \mathrm{~cm}$ in front of the participant. Picture presentation lasted for $37 \mathrm{~min}$ with a short break in the middle of the session to allow for posture adjust-

${ }^{1}$ IAPS numbers of the selected pictures $(n=300)$ are provided upon request by the corresponding author (Tobias.Flaisch@uni-konstanz.de). 
ments. Subjects were instructed to keep their eyes comfortably focused on the fixation cross in the center of the screen and to simply view the pictures.

\section{Apparatus and Data Analysis}

Brain and ocular scalp potential fields were measured with a 256lead geodesic sensor net (GSN 200 v2.0; EGI: Electrical Geodesics, Inc., Eugene, OR), online bandpass filtered from 0.01 to $100 \mathrm{~Hz}$, and sampled at $250 \mathrm{~Hz}$ using Netstation acquisition software and EGI amplifiers. Electrode impedance was kept below $50 \mathrm{k} \Omega$, as recommended for this type of electroencephalogram (EEG) amplifier by EGI guidelines. Data were recorded continuously with the vertex sensor as reference electrode. Continuous EEG data were low-pass filtered at $35 \mathrm{~Hz}$ using a zero-phase forward and reverse digital filter before stimulus synchronized epochs were extracted from $336 \mathrm{~ms}$ before the prime (first) picture until $336 \mathrm{~ms}$ after the offset of the target (second) picture (672 ms) and baseline corrected for prestimulus (20 ms) prime ERP activity.

Data editing and artifact rejection were based on a two-step method for statistical control of artifacts (Junghöfer, Elbert, Tucker, \& Rockstroh, 2000). In a first pass of the data (using the recording reference), sensors contaminated across the session were identified and rejected. Furthermore, sensors containing trial epochs with artifact activity were rejected to avoid contamination when converting the data to an average reference. The rejection of artifact-contaminated trials and sensor epochs was based on the thresholds for a number of statistical parameters (e.g., absolute value over time, standard deviation over time, etc.; cf. Junghöfer et al., 2000). In a second pass, based on the average referenced data, sensors containing artifact-contaminated activity were replaced using spherical interpolation on the basis of all remaining sensors for the given trial.

Average waveforms were calculated for each of the nine experimental cells (three picture categories for the first and second pictures, respectively) for each sensor and participant (see Figure 1b). On average, $26.7 \%$ of trials were excluded during artifact correction. The percentage of rejected trials was comparable across experimental cells (range $=26.0-27.6), F(8,184)=1.7$, n.s., $\varepsilon=.62$. Furthermore, accepted trials contained an average number of 243 sensors and, again, the number of good sensors did not statistically differ between the experimental cells $($ range $=243.2-243.4), F(8,184)=1.4$, n.s., $\varepsilon=0.6$.

\section{Waveform Analysis: Single Sensor Analyses}

In the waveform analyses, each time point and sensor was tested separately using a 3 (first picture: pleasant, neutral, unpleasant) $\times 3$ (second picture: pleasant, neutral, unpleasant) ANOVA. For the first picture, ANOVAs were conducted from picture onset to the end of the second picture presentation (i.e., 0-600 ms). For the second picture, ANOVAs were conducted from the onset to the end of the presentation of the second picture. The waveform analyses served as a means to determine time course and topographical distribution of modulatory effects of first and second picture emotionality.

\section{Area Score Assessment Of Target (Second) Picture Processing}

Building upon the patterns obtained in the previous waveform analyses, subsequent ANOVAs detail the processing of the target (second) picture as a function of its emotionality and the emotionality of the preceding prime (first) picture. Toward this end, mean amplitudes from representative frontal and temporo-oc- cipital sensor clusters (see Figure 2), were averaged over a time interval from 248 to $288 \mathrm{~ms}$ after onset of the target picture. These data were submitted to separate repeated measures ANOVAs including the factors Prime Valence (first picture: pleasant, neutral, unpleasant), Target Valence (second picture: pleasant, neutral, unpleasant), Site (fronto-cental vs. temporo-occipital), and Laterality (left vs. right).

When appropriate, the Greenhouse-Geisser procedure was used to correct for violations of sphericity.

\section{Source Estimation: Current Source Density (CSD)}

The CSD approach recommended by Junghöfer, Elbert, Leiderer, Berg, and Rockstroh (1997), based on a physiological volume conductor model that is well suited for dense array EEG data, was used to determine the calculation of the current source density. In this analysis, a focal generator source is indicated by a sink/source pattern of inward/outward flow of current, which, however, reveals a more complicated distribution of multiple inward and outward currents in the case of activation of simultaneous generator sources. Thus, although this method has limitations in attributions of sink/source patterns to specific neural structures, the technique is sufficiently sensitive to the question to determine the similarity of prime and target emotion effects. To statistically corroborate differential patterns arising from the CSD source estimation, mean values from representative occipito-parietal sensor clusters were averaged over the critical time interval (248-288 $\mathrm{ms}$ ) and submitted to repeated measures ANOVAs including the factors Prime Valence (first picture: pleasant, neutral, unpleasant), Target Valence (second picture: pleasant, neutral, unpleasant), and Laterality (left vs. right).

\section{Results}

\section{Waveform Analyses}

Figure 3 shows the ERP waveforms for a baseline picture, a prime (first) picture, and a target (second) picture at representative frontal, central, and occipital sensor sites. In this figure, picture content varies only for the prime picture (pleasant, neutral, and unpleasant primes), whereas in the baseline and in the target picture interval the stimuli were averaged across all pictures independent of hedonic valence. Thus, the effect of emotionality on the first picture in the pair and its effects on ERPs to a subsequent picture are shown. Figure 4a shows the according topographical distributions of these raw scalp potentials, and the resulting statistical findings of the single sensor waveform analyses are summarized in Figure 4b. For illustrative purposes, both depictions are collapsed into meaningful time bins.

Previous studies found that emotionally arousing (i.e., pleasant and unpleasant) pictures are associated with enhanced posterior negativity compared to neutral images. These effects were replicated in the present study (see Figures 3 and 4b, top panel). When viewing the first picture, the encoding of affective pictures resulted in a pronounced relative negative shift in the ERP over left and right hemispheric temporo-occipital sites, as illustrated by the waveforms observed for a representative left and a right hemispheric temporo-occipital sensor (see Figure 3). Furthermore, due to polarity reversal, a corresponding positive shift was observed over left and right fronto-central sites, again illustrated by two representative left and right hemispheric frontal sensors (see Figure 3). As in previous studies, emotional 


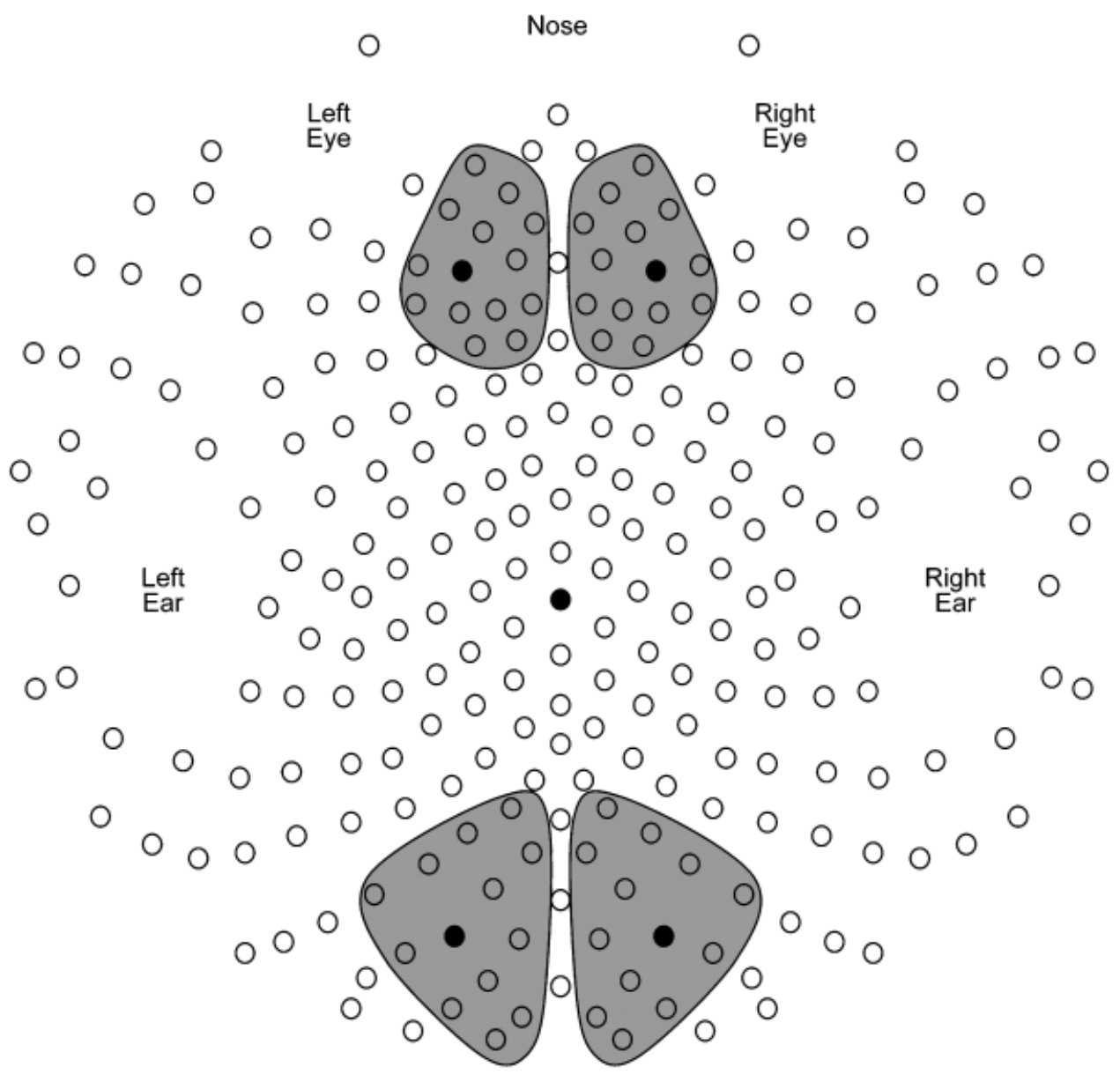

Figure 2. Illustration of the sensor montage of the high-density EEG system. Gray areas indicate sensor clusters included in the conventional ANOVA analysis (EGI sensor numbers: temporo-occipital left: 115, 116, 117, 118, 123, 124, 125, 126, 135, 136, 137, 146, 147; temporo-occipital right: 139, 140,149,150,151, 157, 158, 159, 160, 166, 167, 168, 169; fronto-central left: 22, 23, 26, 27, $28,29,32,33,34,35,36,37,38,39$; fronto-central right: 3, 4, 5, 6, 11, 12, 13, 14, 18, 19, 20, 216, 225, 226). ERP-activity at marked sensors is presented in detail in Figure 3 (EGI 124, 159, 89, 34, 4) and in Figure 6 (EGI 159).

modulation was most apparent for pleasant picture contents (cf. Schupp et al., 2006).

The emotionality of the prime picture also prompted pronounced differences over centro-parietal sensors around 300-500 ms after prime onset (see Figure $4 b$, top panel), and thus extending into the processing period of the following target picture (0-200 ms after target onset). The topography and polarity of this effect resembles the centro-parietal late positive potential (LPP) typically found for emotionally arousing pictures. As shown for a representative central sensor (\#89; see Figure 3), pleasant and unpleasant pictures prompted a relative positivity, compared to neutral pictures in this time window (cf. Schupp et al., 2000).

The hedonic valence of the prime picture also affected ERPs when subsequent pictures were viewed, in a window from 200 to $300 \mathrm{~ms}$ after the onset of the second picture. This effect is illustrated in Figure 4b (top panel): Again, sensors in temporooccipital and fronto-central regions showed distinct main effects for the emotionality of the first picture. In this case, however, as detailed in Figures 3, 5, and 6, the effect was opposite in direction: Whereas a relative negative potential over posterior sites in a window from 200 to 300 was obtained when emotionally arous- ing first pictures were viewed, these pictures elicited a relatively more positive potential in the same time window when a subsequent picture was presented. Furthermore, consistent with a hypothesis that these effects are similar with regard to temporal and spatial appearance, but opposite in direction, the effect on the subsequent picture was most pronounced when the preceding picture was pleasant.

Figure $4 \mathrm{~b}$ (middle panel) indicates the sensors showing a main effect of the emotionality of the second picture (target effect) during its presentation. As found for the first picture, both temporo-occipital and fronto-central sensors were affected by picture emotionality. Figure 6 illustrates that the modulatory differences over temporo-occipital sensors during second picture processing were highly similar to those found during first picture processing, in which enhanced negativity was obtained when viewing emotional, compared to neutral, pictures. Again, the effect was most pronounced for pleasant pictures.

In the exploratory waveform analyses, no interaction effects between the emotionality of first and second pictures were apparent, as indicated in Figure 4b (bottom panel). Thus, the hedonic congruence between the first and second pictures in a pair did not appear to modulate the ERP waveforms. 

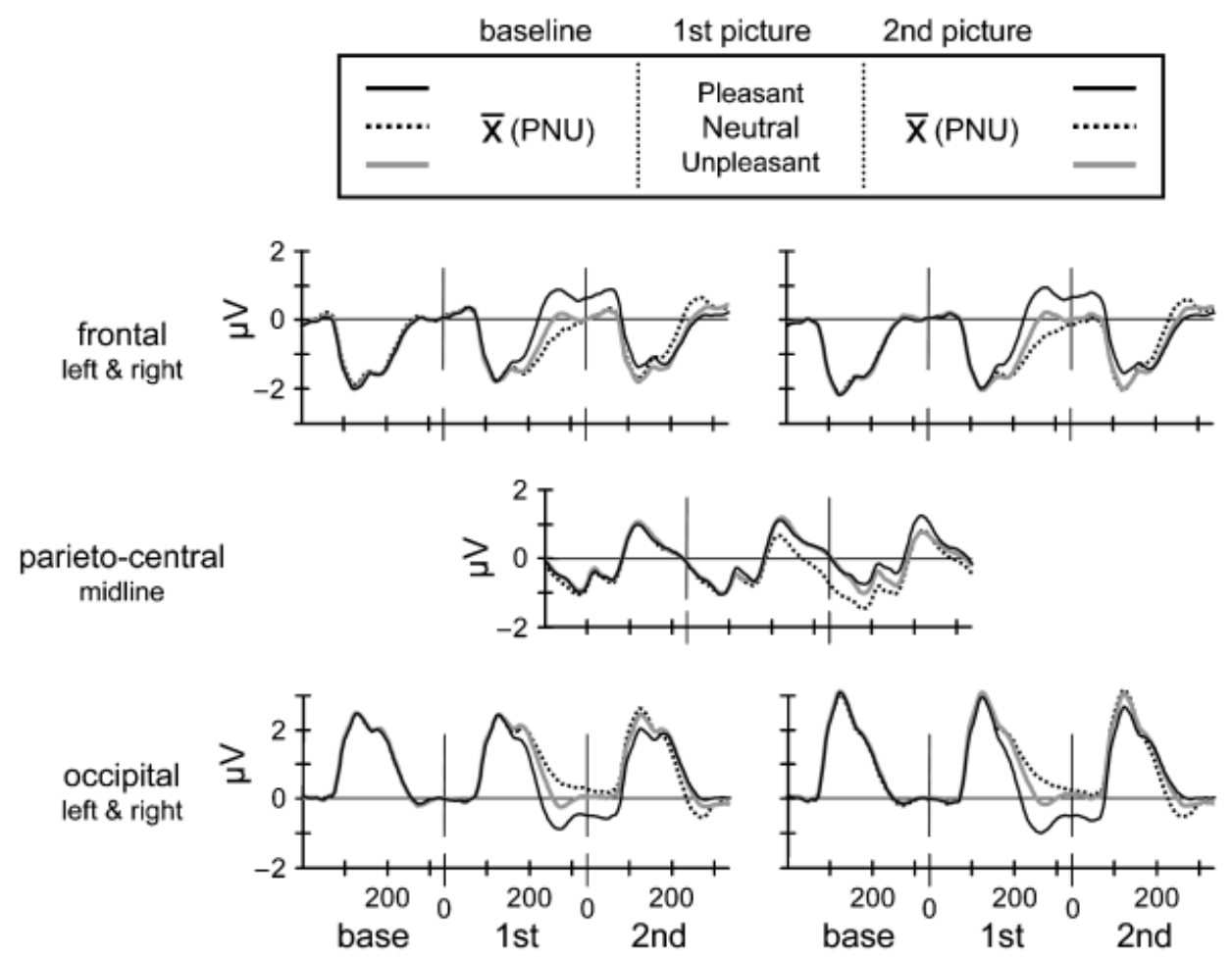

Figure 3. Illustration of the ERP waveforms for a sequence of three consecutive pictures for five representative sensors (see Figure 2). The sequence includes a baseline picture (averaged over valence), followed by illustration of the ERPs elicited for the first and second pictures in a pair. Picture content is varied for the first picture in the pair and averaged across valence for the second picture in a pair. Thus, the main effect of picture emotionality on processing is apparent in the ERPs for the first picture in a pair, and the effects of emotion on subsequent pictures is illustrated in the ERPs for the second picture in the pair.

\section{Area Score Assessment of Target (Second) Picture Processing}

To more precisely determine statistical effects associated with prime and target picture emotionality, the outcome of the single sensor waveform analysis was followed up by conventional ANOVAs. Towards this end, analyses of area scores computed in a time window from 248 to $288 \mathrm{~ms}$ after onset of the second picture were calculated for a group of temporo-occipital and fronto-central sensors. As expected, a main effect of Target Valence was found when processing the second picture in the pair for both temporo-occipital and fronto-central sensors, $F \mathrm{~s}(2,46)=44.2$ and 58.8, $p<.001, \varepsilon=.81$ and .75 . Pleasant and unpleasant target pictures were associated with a more negative-going potential compared to neutral stimuli over temporooccipital sensors, $F(1,23)=59.5$ and $16.8, p<.001$, and a more positive going potential over fronto-central sensors, $F(1,23)=78.6$ and $17.3, p<.001$, both of which were more pronounced for pleasant, compared to unpleasant, target pictures, $F_{\mathrm{s}}(1,23)=45.8$ and $86.5, p<.001$. Figure 6 a illustrates this effect when pictures were preceded by a pleasant, neutral, or unpleasant picture: In each case, emotionally arousing (pleasant or unpleasant) pictures prompted greater occipital negativity than neutral pictures, suggesting that this early modulatory effect reflects current processing requirements.

On the other hand, a main effect of the emotionality of the preceding picture was also found for both temporo-occipital and fronto-central sensors, Prime Valence $F(2,46)=22.0$ and 28.4, $p<.001, \varepsilon=.9$ and .96 . As can be seen in Figure 6b (after rearranging the cells of $6 \mathrm{a}$ ), all pictures preceded by pleasant or unpleasant pictures were associated with relatively less negativity over temporo-occipital sensors, compared to neutral pictures,
$F_{\mathrm{S}}(1,23)=33.3$ and $15.0, p<.001$, as well as less positivity over fronto-central sensors, $F \mathrm{~s}(1,23)=52.7$ and $18.5, p<.001$. Again, both of these effects were more pronounced when pleasant pictures preceded the target, compared to unpleasant contents, $F \mathrm{~s}(1,23)=11.1$ and 10.3 for temporo-occipital and fronto-central sensors, respectively, $p<.01$. A significant interaction of Target Valence and Laterality, $F(2,46)=6.7, p<.01, \varepsilon=.84$, for temporo-occipital sensors suggested that emotional modulation was somewhat more pronounced over the right temporo-occipital region, $F \mathrm{~s}(1,23)>17, p<.001$, although also highly significant over the left temporo-occipital sensor cluster, $F \mathrm{~s}(1,23)>15$, $p<.001$.

\section{Current Source Density}

The comparison of the scalp-recorded difference potential maps (emotional - neutral) suggests that the topographies of the target and prime effects are related but opposite in direction. A more informative test of this notion is provided by the current source density analysis, which emphasizes focal compared to global (widespread and blurred) sources of activation. Focusing on the target emotion effect (see Figure 5b, upper row), the CSD analysis replicated previous findings by revealing bilateral generator structures in posterior regions (Junghöfer et al., 2001). Statistically analyzing these patterns over corresponding occipito-parietal sensor clusters revealed a significant main effect of Target Valence, $F(2,46)=16.8, p<.001, \varepsilon=.67$, indicating that pleasant and unpleasant as compared to neutral target stimuli were estimated by relative negative sources, $F_{\mathrm{s}}(1,23)>15, p<.001$. Interestingly, although less pronounced, a sink/source pattern of opposite direction but closely related topography was seen for 


\section{Scalp potential}

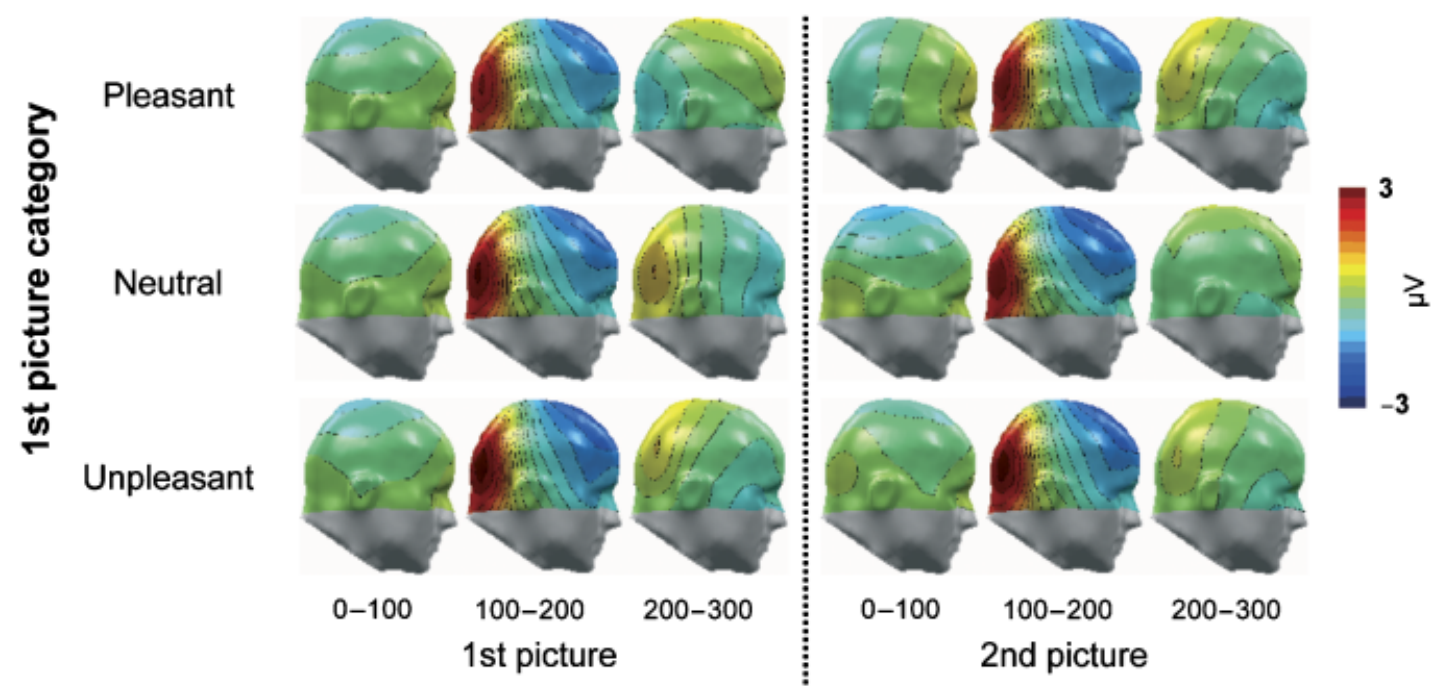

B

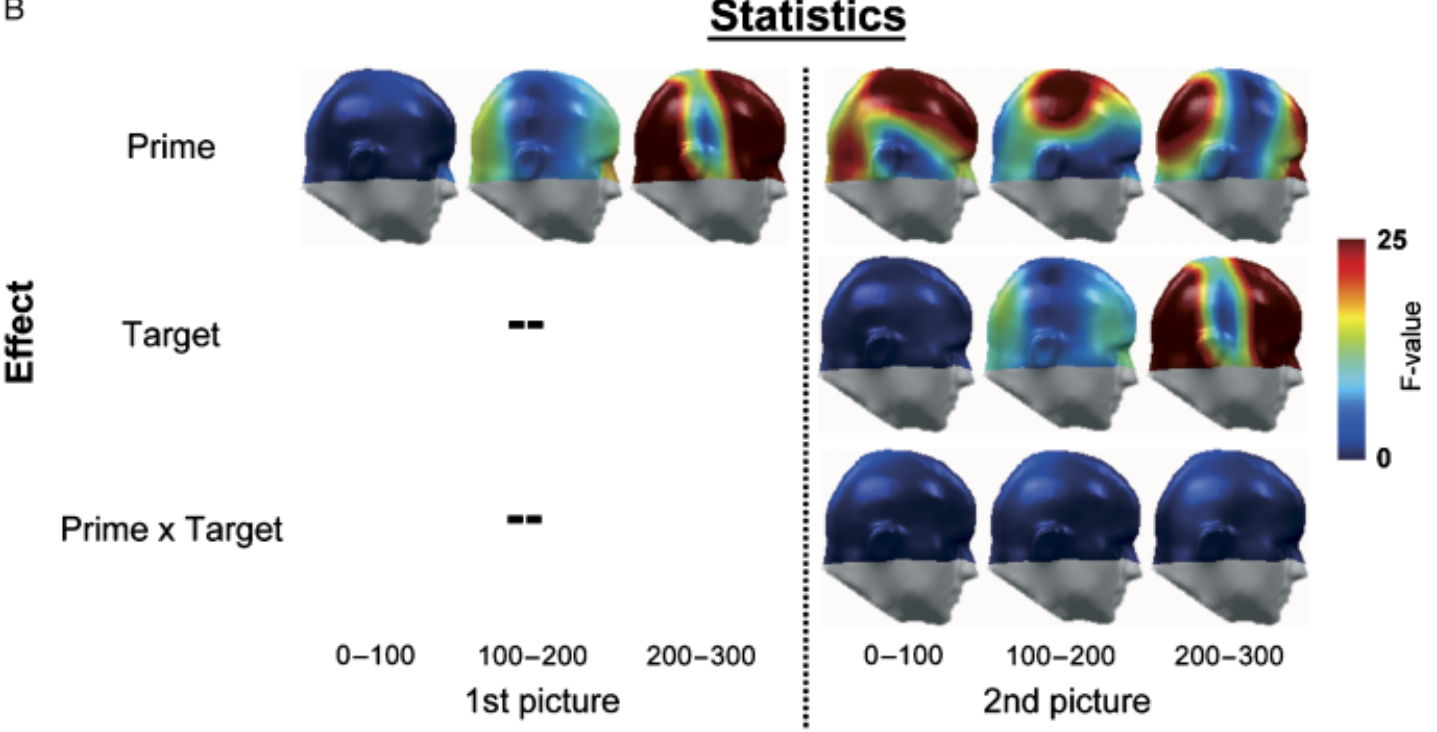

Figure 4. A: Collapsing across meaningful time bins, the topographical distribution of the original scalp potentials is illustrated. As in Figure 3, picture content is varied for the first picture epoch and averaged across valence for the second picture. B: Based on the analysis of all sensors, the outcome of the point-by-point waveform ANOVA is illustrated in terms of $F$ values (again collapsed across meaningful time bins). Note that the effect of emotion on picture processing for the first picture epoch (prime effect) is highly similar to the effect seen when processing the second picture (target effect), reflecting the effects of emotion on current picture processing. In contrast, the effect of emotion on processing of the subsequent picture is reflected in the second picture period in the prime tests.

the CSD analysis of the prime emotion effect, Prime Valence, $F(2,46)=9.6, p<.01, \varepsilon=.77$ (see Figure 5b, lower row). Although not differing from each other, both pleasant and unpleasant prime pictures were associated with a more positivegoing source pattern than neutral primes, $F \mathrm{~s}(1,23)>10, p<.01$.

\section{Control Analyses}

Baseline assessment. In the rapid serial visual presentation paradigm, issues regarding the appropriate baseline often arise. In the current study, the use of the baseline picture, which represented all possible picture contents, is perhaps the best estimate, as effects due to picture content were experimentally controlled (cf. Figure 1). On the other hand, we also assessed effects of other baseline measures to determine the generality of the obtained differences. First, the data were reanalyzed using a baseline time-locked to the onset of the target (second) picture. This analysis replicated the findings reported above. Thus, emotionality of the first picture again affected subsequent picture processing, $F(2,46)=38.6, p<.001, \varepsilon=.91$, with ERPs showing a less negative potential over temporo-occipital sensors when the second picture was preceded by emotional, compared to neutral, contents, and the same pattern with reversed polarity over fronto-central sensors, $F(2,46)=59.5, p<.001, \varepsilon=.84$, both of which were again most accentuated for pleasant contents. 


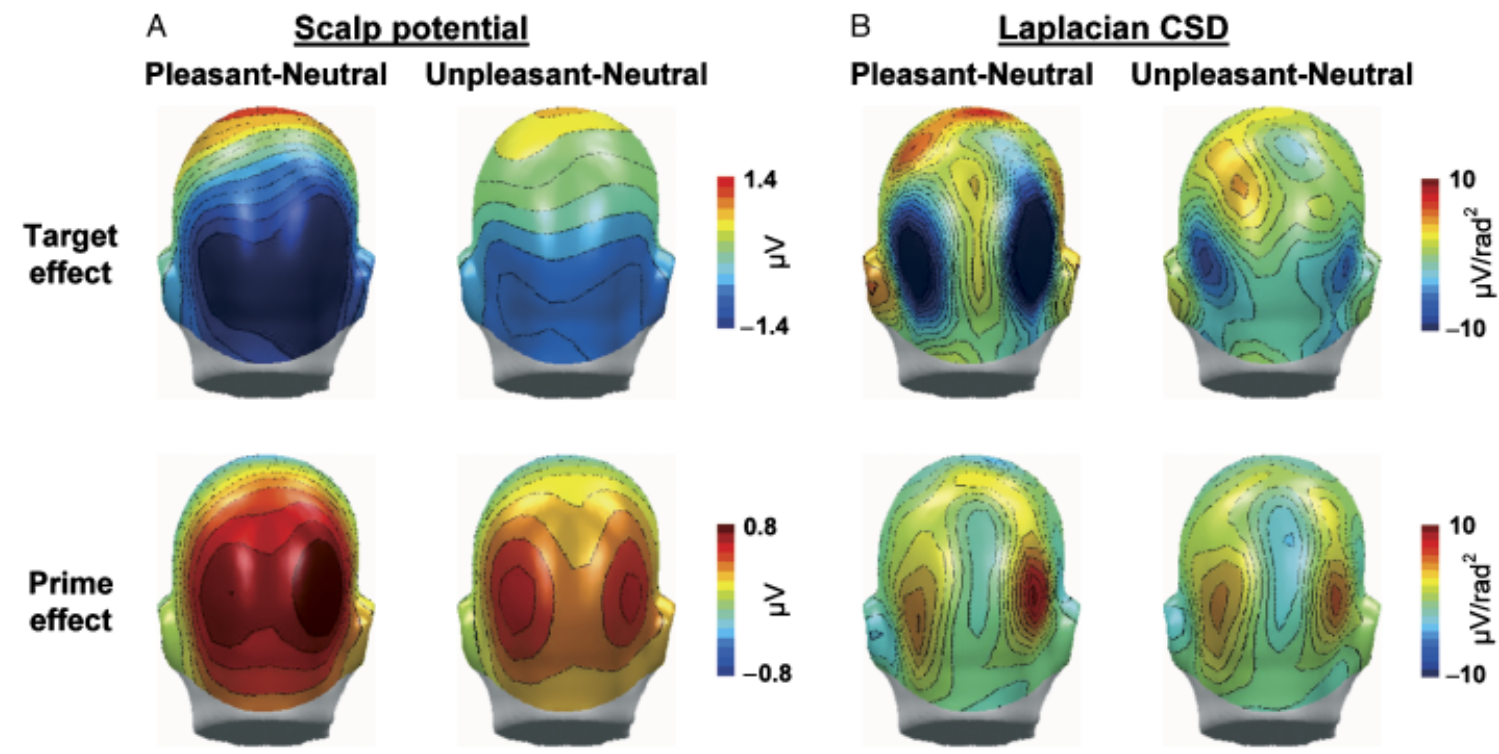

Figure 5. A: Scalp potential maps of the difference waves (pleasant - neutral) and (unpleasant - neutral) for the interval used in statistical analysis (248-288 ms) reveal the topographical distributions of effects of emotion on picture processing. To derive these brain maps, voltages were interpolated to the scalp surface using spherical splines and back-projected to a back view of the model head. Please note the different scales for target and prime effect. B: Topographical distributions of the current source density ( $\sim$ Laplacian) of the difference waves (pleasant - neutral) and (unpleasant - neutral) for the interval used in statistical analysis.

A second approach used a baseline-free estimate of the effects by determining the gradient (slope) of the scalp potential based on the first temporal derivative of the ERP waveform. This procedure in effect constitutes a specific temporal high-pass filter and allows one to compare ERP waveforms with respect to the onset and the steepness of the comprised components independent of any baseline correction. ${ }^{2}$ Analysis of the gradient measure supported the findings of the main analysis. Most importantly, the gradient analysis again resulted in a significant effect of emotionality of the first picture on subsequent processing for both temporo-occipital and fronto-central sensors, $F \mathrm{~s}(2,46)=18.9$ and $27.8, p<.001, \varepsilon=.97$ and .98 , in which the ERP gradient in the time window of 180-220 ms after the onset of the second picture was less steep when preceded by emotional compared to neutral images, again most accentuated for pleasant contents.

Residual processing effects. In rapid serial picture presentation, ERP activity may extend into the processing window of a subsequently presented picture, leading to a challenge in distinguishing whether effects of a prime stimulus reflect altered target processing or, alternatively, may be attributed to sustained prime picture processing. Specifically, the concern is whether the prime effects in the time interval of 200 to $300 \mathrm{~ms}$ after target onset are related to sustained emotional processing of prime pictures as indexed by late positive potentials. To assess this hypothesis, we first scored the ERP activity in a centro-parietal cluster (see Supplementary Figure 3; see Supplementary Material, below) in a time window from 350 to $450 \mathrm{~ms}$ after onset of the prime picture, corresponding to 16-116 ms after target onset (see also Schupp et al., 2007b). A significant main effect of Prime Valence

${ }^{2}$ Specifically, in a first step, maximal modulation of the first derivative as a function of prime and target pictures was determined to occur in a time interval of 180-220 ms by means of single waveform analysis. In a second step, the mean gradient values were calculated in frontal and temporo-occipital sensor clusters in the resulting time interval and submitted to repeated measures ANOVA. was observed, $F(2,46)=35.3, \quad p<.001, \varepsilon=.61$. Pleasant $(M=-0.3 \mu \mathrm{V})$ and unpleasant $(M=-0.3 \mu \mathrm{V})$ prime pictures were associated with an enhanced positive-going potential compared to neutral stimuli $(M=-0.7 \mu \mathrm{V}), F_{S}(1,23)=33.7$ and $45.2, p<.001$, respectively, and did not differ from each other. Neither the main effect of Target Valence nor the interaction of Prime Valence $\times$ Target Valence was significant, suggesting that this effect differed from later ERP modulations.

A second test concerns whether the anterior and posterior sensor clusters, which are interpreted as reflecting changes in target processing due to prime emotionality, already show similar ERP modulation during the LPP time window. Separate ANOVAs for anterior and posterior sensor clusters revealed Prime Valence effects, $F s(2,46)>18, \varepsilon=.84$ and $=.91, p<.001$, but the modulation was specific to pleasant picture processing, $F s(1,23)>22, p<.001$, and opposite in polarity to that found in the 248-288-ms time window. These differences in topography and pattern of modulation suggest that the prime effects on later target processing (e.g., 248-288-ms window) are not strongly determined by superposition of late ERP activity associated with prime picture processing.

\section{Discussion}

Previous studies utilizing rapid picture presentations have found that emotionally significant stimuli are discriminated from neutral pictures, with pleasant and unpleasant pictures prompting increased occipital negativity in a $150-300-\mathrm{ms}$ time window (cf. Junghöfer et al., 2001; Schupp et al., 2003). These results were replicated in the present study: Pictures that were pleasant or unpleasant prompted greater occipital negativity than neutral pictures, despite the rapid rate of picture presentation. More importantly, the present study found that the magnitude of posterior negativity for a target picture varies systematically as a function of the preceding prime. When a prime picture was emotional (and itself eliciting heightened posterior negativity), posterior negativity of the current target picture was reduced. 
A

Target Category

- Pleasant

...... Neutral

Unpleasant
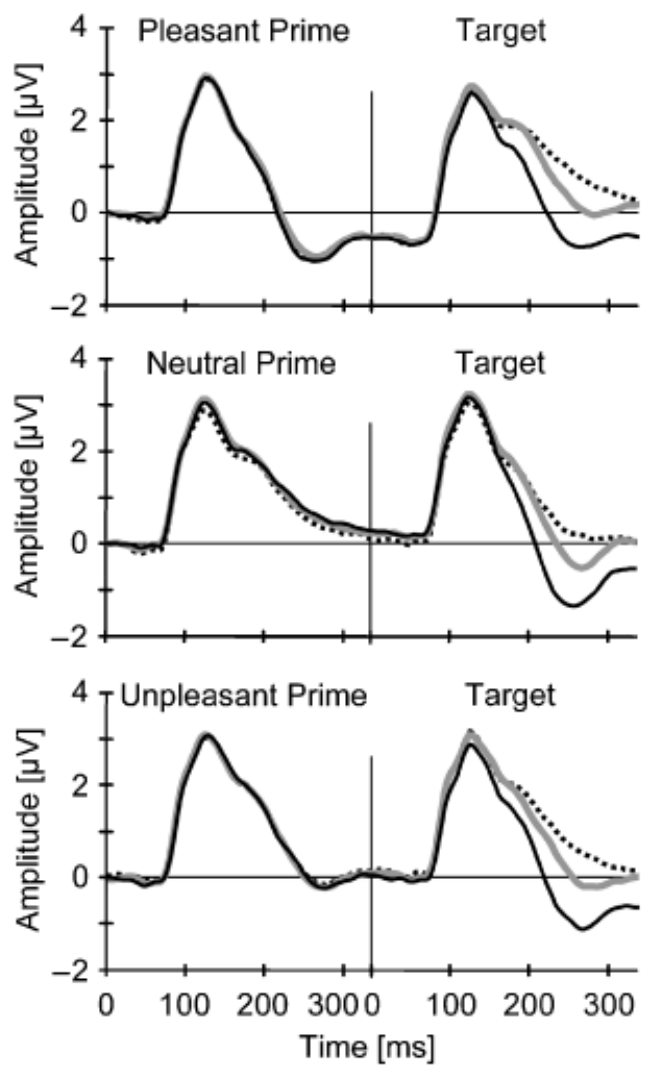

B

Prime Category

- Pleasant

...... Neutral

Unpleasant
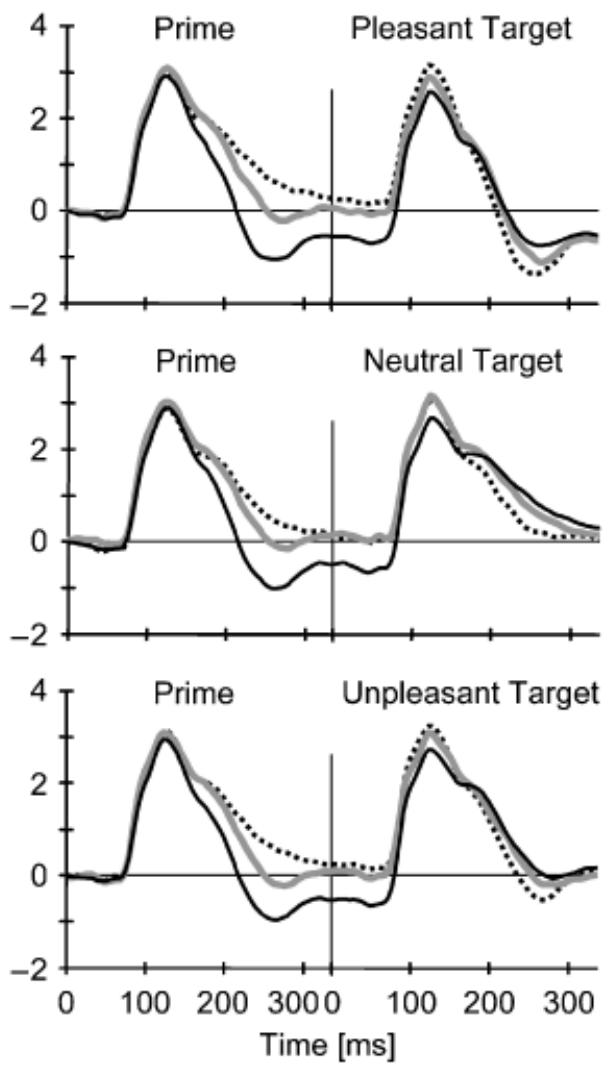

Figure 6. ERP waveforms for a representative right-occipital sensor (159, see Figure 2) illustrating the effects of emotion during picture processing (A) and on subsequent picture processing (B) for all cells in the design.

This effect of an emotional prime was identical regardless of whether the target picture was pleasant, neutral, or unpleasant. Thus, the data reveal no evidence for affective priming in the perceptual domain. Rather, irrespective of whether the hedonic valence of the prime matched or mismatched the target, occipital negativity was decreased if the prime picture was affectively engaging. Taken together, these results suggest that enhanced processing of affective primes diminishes subsequent target processing, regardless of the hedonic content of the target.

The novel finding of the present study is that current target processing is affected not only by the emotional content of the target image but also systematically varies with the emotional content of the preceding prime picture. When a preceding picture was emotional, ERPs during viewing of the subsequent picture were reduced in the same time window (e.g., 200-300 ms) and over the same sensors (e.g., temporo-occipital and fronto-central). One interpretation of these findings is that selective emotion processing reduces or interferes with the recognition and categorization of subsequent stimuli, regardless of their hedonic content. Thus, these data may provide ERP evidence for what Most and colleagues (2005) recently called "emotion-induced blindness," in which the detection of target stimuli suffers from following an affectively engaging picture.

In the RSVP paradigm, effects of a preceding stimulus on a later target are often attributed to competition and sharing of processing resources (Potter, Staub, \& O'Connor, 2002; Shapiro et al., 1997). In this view, if a preceding stimulus captures pro- cessing resources, it interferes with processing of subsequently presented stimulus materials. The notion of interference and competition for resources has been examined in a number of paradigms in which processing is probed when emotional and non-emotional stimuli are presented concurrently (Anderson, Christoff, Panitz, De Rosa, \& Gabrieli, 2003; Pessoa, McKenna, Gutierrez, \& Ungerleider, 2002; Vuilleumier, Armony, Driver, \& Dolan, 2001). For instance, a recent ERP study observed that a frontal positivity $(100 \mathrm{~ms})$ elicited by fearful faces was only present when the faces were presented at attended locations whereas it was absent when fearful faces were presented at nonattended locations (Holmes, Vuilleumier, \& Eimer, 2003). Similar findings have been observed in another ERP study exploring the processing of emotional facial expressions (Pourtois, Grandjean, Sander, \& Vuilleumier, 2004). A more recent study demonstrated that interference of emotion processing by a competing task extends to the domain of feature-based attention. Specifically, taxing perceptual resources by a competing primary visual attention task markedly attenuated the EPN difference associated with emotional compared to neutral IAPS picture contents (Schupp et al., 2007a). Thus, selective emotion processing might be attenuated by concurrently presented stimuli commanding processing resources.

Although broadly consistent with these findings, the present study design differs in several ways from these studies that merits reemphasis. Rather than assessing effects due to concurrent processing, the present results suggest that processing resources al- 
located to target pictures systematically vary with the amount of processing resources captured by preceding emotional prime stimuli. Furthermore, whereas previous studies assessed competition effects mainly by introducing an explicit attention task, modulatory effects of affective pictures on subsequent processing were found here in the absence of any active task instruction, presumably reflecting the implicit capture of attention by emotional cues (cf. Öhman, Flykt, \& Lundqvist, 2000).

The presentation of pictures in a rapid and continuous stream introduces caveats and limitations regarding the interpretation of ERP components. For instance, rather than reflecting changes in target picture processing, the reported ERP differences may reflect the sustained processing of the prime picture. However, a lingering LPP effect for emotional prime pictures, that is, enhanced centro-parietal positive potentials accompanied by an enhanced negativity at temporo-occipital sites (cf. Schupp et al., 2007a, 2007b), was not observed. In fact, the pattern elicited by emotional prime pictures, that is, a reduced temporo-occipital negativity, was opposite in polarity.

Of course, it is possible that the neuronal processes engaged in target picture processing shift the topography of scalp-recorded brain potential differences associated with emotional prime picture processing. Although this is theoretically possible, aspects of the present data appear inconsistent with this notion. Specifically, although pleasant and unpleasant pictures elicited comparable LPP amplitudes, the occipital negativity found for emotional targets and its reduction as a function of being preceded by an emotional prime was markedly more pronounced for pleasant pictures. Furthermore, a follow-up study, in which picture presentation time was longer $(660 \mathrm{~ms})$, revealed a qualitatively similar effect of emotional primes (Stockburger, Flaisch, Bublatzky, Schmälzle, \& Schupp, 2007). In that study, the emotional target and prime effects were observed with striking parallels regarding topographical appearance but with opposite polarity for both early and late ERP measures.

Current source density analysis provides further support for the interpretation that prime and target effects are linked to similar neural generator structures. The CSD of the difference maps (emotional - neutral) showed a very similar topography of the sink/source patterns, again in opposite directions for the prime and target effects (see Figure 5b). Overall, although not definitive, the current data seem consistent with the notion that emotionally arousing pictures not only impact current picture processing but also modulate subsequent pictures in rapid serial presentations. The systematic variation of presentation times in future studies may provide further information to more precisely determine the locus of interference at the level of specific processing stages.

A further challenge introduced by the RSVP paradigm concerns estimating baseline activity (cf. Urbach \& Kutas, 2006). The present study was successful in achieving comparable ERP baseline activity associated with the images preceding the prime pictures by insuring that all picture contents preceded these important stimuli. On the other hand, ERP activity preceding the target picture was markedly different as a function of prime content. As a conservative approach, the main results for the target picture were reported using ERP baseline activity preced- ing the prime picture. Nonetheless, follow-up tests assessed these effects using a baseline immediately preceding target presentation, which augmented, rather than eliminated, the effects of an emotional prime. Moreover, a baseline-free gradient measure was applied that also resulted in strong effect of emotional prime on the target ERPs. Overall, converging evidence supports the interpretation that negativity over occipital sensors in the 200300-ms window reflects current picture processing, with enhanced negativity when selective processing is underway and decreased negativity when encoding is more impoverished.

The present data provided no evidence for affective priming effects in the first $300 \mathrm{~ms}$ of perceptual processing. Specifically, the processing of pleasant or unpleasant cues was not differentially affected as a function of whether the preceding picture was the same in hedonic valence. Rather, effects were most pronounced for all pictures when a pleasant picture preceded a target. Previous studies have suggested that this difference is mediated primarily by pictures of erotic content (Codispoti et al., 2006a; Schupp et al., 2006). One hypothesis is that features of erotic pictures are easier to recognize and categorize, prompting more efficient encoding and categorization, particularly at these rapid rates. This assumption is consistent with recent data that found that simple figure-ground pictures also prompt relative negativity over temporo-occipital sensors beginning around 150 $\mathrm{ms}$ after picture onset, compared to pictures depicting more complex scenes, suggesting that the early occipital negativity may reflect, in part, the ease with which stimulus features are matched to memory representations (Bradley, Hamby, Löw, \& Lang, 2007).

In summary, previous studies have observed facilitated processing of emotionally arousing cues when stimuli are presented in a rapid and continuous stream. These findings were extended here by demonstrating that processing emotionally arousing pictures affects the processing of subsequently presented pictures, irrespective of whether these pictures depicted emotional or neutral contents. Future studies need to determine whether resource competition among successively presented pictures provides a reasonable interpretation of the results.

\section{Supplementary Material}

The following supplementary material is available for this article (all Figures provided in PDF format):

Figure S1. Vertcial and horizontal EOG.

Figure S2. Original and difference wave topographies of the analyzed time interval.

Figure S3. Illustration of sensor cluster used for LPP analysis.

This material is available as part of the online article from: http://www.blackwell-synergy.com/doi/abs/10.1111/j.1469-

8986.2007.00600.x (This link will take you to the article abstract).

Please note: Blackwell Publishing is not responsible for the content or functionality of any supplementary materials supplied by the authors. Any queries (other than missing material) should be directed to the corresponding author for the article.

\section{REFERENCES}

Anderson, A. K., Christoff, K., Panitz, D., De Rosa, E., \& Gabrieli, J. D. (2003). Neural correlates of the automatic processing of threat facial signals. Journal of Neuroscience, 23, 5627-5633.
Avero, P., \& Calvo, M. G. (2006). Affective priming with pictures of emotional scenes: The role of perceptual similarity and category relatedness. Spanish Journal of Psychology, 9, 10-18. 
Bradley, M. M., Hamby, S., Löw, A., \& Lang, P. J. (2007). Brain potentials in perception: Picture complexity and emotional arousal. Psychophysiology, 44, 364-373.

Chun, M. M., \& Potter, M. C. (1995). A two-stage model for multiple target detection in rapid serial visual presentation. Journal of Experimental Psychology: Human Perception and Performance, 21, 109127.

Codispoti, M., Ferrari, V., \& Bradley, M. M. (2006a). Repetitive picture processing: Autonomic and cortical correlates. Brain Research, 1068, 213-220.

Codispoti, M., Ferrari, V., \& Bradley, M. M. (2007). Repetition and event-related potentials: Distinguishing early and late processes in affective picture perception. Journal of Cognitive Neuroscience, 19, $577-586$.

Codispoti, M., Ferrari, V., Junghöfer, M., \& Schupp, H. T. (2006b). The categorization of natural scenes: Brain attention networks revealed by dense sensor ERPs. NeuroImage, 32, 583-591.

Cuthbert, B. N., Schupp, H. T., Bradley, M. M., Birbaumer, N., \& Lang, P. J. (2000). Brain potentials in affective picture processing: Covariation with autonomic arousal and affective report. Biological Psychology, 52, 95-111.

Holmes, A., Vuilleumier, P., \& Eimer, M. (2003). The processing of emotional facial expression is gated by spatial attention: Evidence from event-related brain potentials. Cognitive Brain Research, 16, 174-184.

Intraub, H. (1999). Understanding and remembering briefly glimpsed pictures: Implications for visual scanning and memory. In V. Coltheart (Ed.), Fleeting memories: Cognition of brief visual stimuli (pp. 47-70). Cambridge, MA: MIT Press.

Junghöfer, M., Bradley, M. M., Elbert, T. R., \& Lang, P. J. (2001). Fleeting images: A new look at early emotion discrimination. Psychophysiology, 38, 175-178.

Junghöfer, M., Elbert, T., Leiderer, P., Berg, P., \& Rockstroh, B. (1997). Mapping EEG potentials on the surface of the brain: A strategy for uncovering cortical sources. Brain Topography, 9, 203-217.

Junghöfer, M., Elbert, T., Tucker, D. M., \& Rockstroh, B. (2000). Statistical control of artifacts in dense array EEG/MEG studies. Psychophysiology, 37, 523-532.

Klauer, K. C., \& Musch, J. (2003). Affective priming: Findings and theories. In J. Musch \& K. C. Klauer (Eds.), The psychology of evaluation: Affective processes in cognition and emotion (pp. 7-49). Mahwah, NJ: Erlbaum.

Lang, P. J., Bradley, M. M., \& Cuthbert, B. N. (1997). Motivated attention: Affect, activation, and action. In P. J. Lang, R. F. Simons, \& M. Balaban (Eds.), Attention and emotion: Sensory and motivational processes (pp. 97-135). Mahwah, NJ: Erlbaum.

Lang, P. J., Bradley, M. M., \& Cuthbert, B. N. (2005). International affective picture system (IAPS): Affective ratings of pictures and instruction manual. Technical Report A-6. Gainesville, FL: University of Florida

Most, S. B., Chun, M. M., Widders, D. M., \& Zald, D. H. (2005). Attentional rubbernecking: Cognitive control and personality in emotion-induced blindness. Psychonomic Bulletin \& Review, 12, 654 661
Öhman, A., Flykt, A., \& Lundqvist, D. (2000). Unconscious emotion: Evolutionary perspectives, psychophysiological data and neuropsychological mechanisms. In R. D. Lane \& L. Nadel (Eds.), Cognitive neuroscience of emotion (pp. 296-327). New York: Oxford University Press

Pessoa, L., McKenna, M., Gutierrez, E., \& Ungerleider, L. G. (2002). Neural processing of emotional faces requires attention. Proceedings of the National Academy of Sciences, USA, 99, 11458-11463.

Potter, M. C. (1999). Understanding sentences and scenes: The role of conceptual short-term memory. In V. Coltheart (Ed.), Fleeting memories: Cognition of brief visual stimuli (pp. 13-46). Cambridge, MA: MIT Press.

Potter, M. C., Staub, A., \& O'Connor, D. H. (2002). The time course of competition for attention: Attention is initially labile. Journal of Experimental Psychology: Human Perception and Performance, 28, $1149-1162$.

Potts, G. F., \& Tucker, D. M. (2001). Frontal evaluation and posterior representation in target detection. Cognitive Brain Research, 11, 147-156.

Pourtois, G., Grandjean, D., Sander, D., \& Vuilleumier, P. (2004). Electrophysiological correlates of rapid spatial orienting towards fearful faces. Cerebral Cortex, 14, 619-633.

Schupp, H. T., Cuthbert, B. N., Bradley, M. M., Cacioppo, J. T., Ito, T., \& Lang, P. J. (2000). Affective picture processing: The late positive potential is modulated by motivational relevance. Psychophysiology, $37,257-261$

Schupp, H. T., Junghöfer, M., Weike, A. I., \& Hamm, A. O. (2003). Emotional facilitation of sensory processing in the visual cortex. Psychological Science, 14, 7-13.

Schupp, H. T., Stockburger, J., Bublatzky, F., Junghöfer, M., Weike, A. I., \& Hamm, A. O. (2007a). Explicit attention interferes with selective emotion processing in human extrastriate cortex. BMC Neuroscience, $8,16$.

Schupp, H. T., Stockburger, J., Codispoti, M., Junghöfer, M., Weike, A. I., \& Hamm, A. O. (2006). Stimulus novelty and emotion perception: The near absence of habituation in the visual cortex. NeuroReport, 17, 365-369.

Schupp, H. T., Stockburger, J., Codispoti, M., Junghöfer, M., Weike, A. I., \& Hamm, A. O. (2007b). Selective visual attention to emotion. The Journal of Neuroscience, 27, 1082-1089.

Shapiro, K., Arnell, K. M., \& Raymond, J. E. (1997). The attentional blink. Trends in Cognitive Sciences, 1, 291-296.

Stockburger, J., Flaisch, T., Bublatzky, F., Schmälzle, R., \& Schupp, H. T. (2007). Sequential picture processing: An ERP-Analysis of affective primes and targets [Abstract]. Psychophysiology, 44 (Suppl. 1), S108.

Urbach, T. P., \& Kutas, M. (2006). Interpreting event-related brain potential (ERP) distributions: Implications of baseline potentials and variability with application to amplitude normalization by vector scaling. Biological Psychology, 72, 333-343.

Vuilleumier, P., Armony, J. L., Driver, J., \& Dolan, R. J. (2001). Effects of attention and emotion on face processing in the human brain: An event-related fMRI study. Neuron, 30, 829-841. 